\title{
Theta synchronizes the activity of medial prefrontal neurons during learning
}

\author{
Rony Paz, ${ }^{1}$ Elizabeth P. Bauer, ${ }^{2}$ and Denis Parée, \\ ${ }^{1}$ Department of Neurobiology, The Weizmann Institute of Science, Rehovot 76100, Israel; ${ }^{2}$ Center for Molecular and Behavioral \\ Neuroscience, Rutgers, The State University of New Jersey, Newark, New Jersey 07102, USA
}

\begin{abstract}
Memory consolidation is thought to involve the gradual transfer of transient hippocampal-dependent traces to distributed neocortical sites via the rhinal cortices. Recently, medial prefrontal (mPFC) neurons were shown to facilitate this process when their activity becomes synchronized. However, the mechanisms underlying this enhanced synchrony remain unclear. Because the hippocampus projects to the mPFC, we tested whether theta oscillations contribute to synchronize mPFC neurons during learning. Thus, we obtained field (LFP) and unit recordings from multiple mPFC sites during the acquisition of a trace-conditioning task, where a visual conditioned stimulus (CS) predicted reward delivery. In quiet waking, the activity of mPFC neurons was modulated by theta oscillations. During conditioning, CS presentation caused an increase in MPFC theta power that augmented as the CS gained predictive value for reward delivery. This increased theta power coincided with a transient theta phase locking at distributed mPFC sites, an effect that was also manifest in the timing of mPFC unit activity. Overall, these results show that theta oscillations contribute to synchronize neuronal activity at distributed mPFC sites, suggesting that the hippocampus, by generating a stronger theta source during learning, can synchronize mPFC activity, in turn facilitating rhinal transfer of its activity to the neocortex.
\end{abstract}

It is widely believed that the hippocampus plays a time-limited role in the formation of declarative memories, because hippocampal lesions produce a temporally graded amnesia for recently learned events (Zola-Morgan and Squire 1986; Kim and Fanselow 1992). It is thought that remote memories are progressively transferred from the hippocampus to the neocortex for long-term storage (Wiltgen et al. 2004). This view is based on the observation that the retrieval of recent versus remote spatial memories produces inverse gradients of activity in the hippocampus and neocortex, including the medial prefrontal cortex (mPFC) (Bontempi et al. 1999).

Consistent with this, much evidence implicates the mPFC in the consolidation of hippocampal-dependent memories. In trace-conditioning tasks for instance, intra-mPFC injections of NMDA antagonists within 2 wk after training impair long-term recall (Takehara-Nishiuchi et al. 2006) and mPFC lesions cause a severe deficit when performed weeks after training, but not immediately after (Takehara et al. 2003). Moreover, expression of the immediate early gene zif268 is enhanced in the mPFC by remote, but not recent contextual memory tests (Frankland et al. 2004). Last, human functional imaging studies have revealed that as the retention interval increases, memory retrieval produces progressively stronger activations in the $\mathrm{mPFC}$, whereas the opposite is seen in the hippocampus (Takashima et al. 2006).

Overall, these data suggest that shortly after training, memories become gradually independent of the hippocampus and that, in parallel, they progressively become more dependent on mPFC activity. Unfortunately, scant data are available regarding the mechanisms underlying this transformation. It is known that most hippocampal-neocortical connections are indirect and involve the rhinal cortices (for review, see Burwell and Witter 2002). However, the mPFC is an exception to this rule. Indeed, in rats, cats, and monkeys, the $\mathrm{mPFC}$ receives a monosynaptic projection from the temporal portion of the hippocampus (CA1 and

${ }^{3}$ Corresponding author.

E-mail pare@axon.rutgers.edu; fax (973) 353-1255.

Article is online at http://www.learnmem.org/cgi/doi/10.1101//m.932408. subiculum) (Swanson 1981; Irle and Markowitsch 1982; Ferino et al. 1987; Jay and Witter 1991; Hirose et al. 1992; Barbas and Blatt 1995; Thierry et al. 2000), and this pathway is strong enough to entrain the firing of mPFC neurons to hippocampal theta oscillations (Jones and Wilson 2005a,b; Siapas et al. 2005). In turn, the mPFC is in a key position to influence rhinal transfer of hippocampal outputs, because it projects to the perirhinal and entorhinal cortices (Room et al. 1985; Sesack et al. 1989; Hurley et al. 1991; Apergis-Schoute et al. 2006).

Recently, we have obtained evidence that the mPFC can facilitate hippocampo-neocortical interactions during the acquisition of a trace-conditioning task by enhancing rhinal transfer of hippocampal impulses to the neocortex (Paz et al. 2007). Key to this effect was an enhanced synchrony in the activity of mPFC neurons in relation to the conditioned stimuli that predicted rewards. However, the mechanisms underlying this increased synchrony remained unclear. The present study was designed to test the hypothesis that theta oscillations contribute to synchronize the activity of $\mathrm{mPFC}$ neurons.

\section{Results}

Using an array of high-impedance microelectrodes (Fig. 1A), we have recorded the activity of mPFC neurons $(n=125)$ in quiet waking and during the acquisition of a trace-conditioning task in three cats. These mPFC neurons included infralimbic (IL, $n=76$ ) and prelimbic (PL, $n=49$ ) cells. The local field potentials (LFPs) picked up by the same electrodes were also monitored. Theta oscillations were isolated by digital filtering of the LFPs (4.5-10 $\mathrm{Hz}$; see Materials and Methods). Below, we will compare the relationship between theta oscillations and unit activity in spontaneous waking conditions and in a learning context. This report only includes neurons that were histologically confirmed to be located in the regions of interest (Fig. 1B).

\section{Spontaneous theta oscillations in quiet waking}

During stimulation-free epochs of quiet waking, theta oscillations of continuously varying amplitude were observed (Fig. 2). 
A
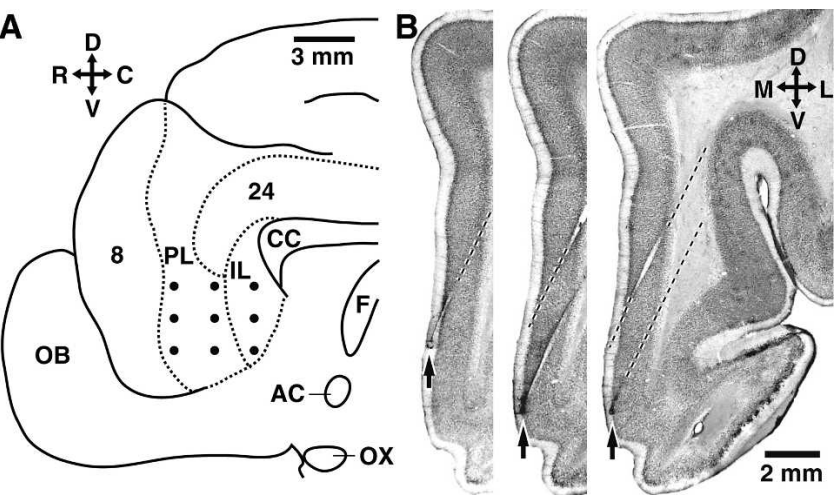

Figure 1. Recording method and histological identification of mPFC recording sites. $(A)$ Scheme showing the midline of the cat brain. Dots indicate intended position of microelectrodes in the mPFC. (B) Histological verification of recording sites. Three adjacent coronal brain sections showing the trajectory of two microelectrodes (dashed lines) as well as the location of electrolytic lesions (arrows) performed at the end of the experiments to mark the position of the microelectrode tips in the MPFC. The orientation of $A$ and $B$ is indicated by crosses where R, C, D, V, M, and $\mathrm{L}$ stand for rostral, caudal, dorsal, ventral, medial, and lateral, respectively. (AC) Anterior commissure; (CC) corpus callosum; (F) fornix; (IL) infralimbic region; $(\mathrm{OB})$ olfactory bulb; (OX) optic chiasma; (PL) prelimbic region.

At times, the theta oscillations seen at different mPFC sites were synchronized (Fig. 2A), but usually they were not (Fig. 2B). To examine the impact of these spontaneous theta oscillations on the activity of mPFC neurons, we first compared the firing rates of mPFC cells during epochs of low vs. high theta power in the LFPs recorded locally. To this end, we measured theta power in 1-sec time windows, and correlated instantaneous firing rate and local theta power for all available cells. As shown in Figure 3A, the firing rates of $\mathrm{mPFC}$ cells showed little correlation to local theta power $(r=0.03, P=0.119)$. Similar findings were obtained when windows of shorter or longer durations were used.

Next, we tested whether the firing rates of mPFC cells were affected by the degree of synchrony of theta oscillations at multiple mPFC sites during periods of high theta power. To this end, in periods of high theta power, we identified the maxima and minima of theta cycles (Fig. 3B1). Maxima and minima points were assigned zero and pi values, the signal was linearly interpolated between them, and then cosined. This created a pure phase signal with standardized amplitude varying between -1 and 1 , independently of the actual theta amplitude (Fig. 3B2). This transformation was performed on all simultaneously recorded LFPs (Fig. 3B3) and the signal averaged (Fig. 3B4). Because these are pure phase signals, moment-to-moment variations in amplitude seen as a result averaging reflect changes in theta synchrony across the simultaneously recorded mPFC sites. Theta synchrony was then quantified by computing the root mean square (RMS) of the signal (Fig. 3B5). As shown in Figure 3C, there was no relationship between firing rates and theta synchrony $(r=-0.04$, $P=0.365)$.

Although these analyses indicate that variations in the amplitude or synchrony of spontaneous theta oscillations are not associated with parallel shifts in the firing rates of mPFC cells, it is possible that theta oscillations affect the temporal structure of their activity. To test this, we divided periods of quiet waking in 3 -sec windows and measured theta power in each. We then computed peri-event histograms (PEHs) of mPFC unit discharges using the positive peaks of theta oscillations as temporal references, separately using windows with the $50 \%$ lowest (Fig. 4 A) vs. $50 \%$ highest theta power (Fig. 4B). The magnitude of theta-related firing was then quantified by computing a rhythmicity index (RI) for the PEHs based on epochs of low vs. high theta power. To compute RIs, we measured the maximal peak to trough difference found within half a cycle of the three central peaks (six measures total), averaged these values, and divided the result by the mean of the entire histogram to normalize for firing rate. To assess significance, we constructed $100 \mathrm{PEHs}$ of unit discharges using the same spike trains, but with respect to random times. The actual PEH was considered significant if its RI was higher than $95 \%$ of the RIs based on randomly generated PEHs.

This analysis revealed that although theta oscillations do not produce consistent changes in firing rates, they have a significant impact on the temporal structure of their activity. Indeed, the RIs computed on unit activity during periods of high theta power were significantly larger (high, $2.93 \pm 0.28$; low, $2.37 \pm 0.20$; paired $t$-test, $P<0.03)$. Moreover, a higher proportion of RIs reached significance when computed on periods of high $(34 \%)$ vs. low $(23 \%)$ theta power (Fisher exact, $P<0.03$; $n=125)$. In addition, this analysis revealed that most mPFC cells tended to fire during the negative phase of the local theta. This point is evident in the inset of Figure $4 \mathrm{~B}$ that plots the frequency distribution of peak times among our sample of $\mathrm{mPFC}$ cells.

\section{Learning-related changes in theta power}

To examine how theta oscillations affect mPFC activity in a learning context, the animals were trained on a traceconditioning task (Fig. 5A). We first describe this task and then consider how theta activity fluctuated as a function of learning. In our trace-conditioning task, the animals were presented a 1.5sec visual conditioned stimulus (CS), followed by a delay period of the same duration, and then by the delivery of a liquid reward. The duration of intertrial intervals varied randomly (30-90 sec). The CS was a global change in the illumination of a computer screen that encompassed most of the visual field of the animals. The animals underwent one training session per day (38-93 trials/session) over a period of $9 \mathrm{~d}$. Evidence that the animals gradually acquired the predictive value of the CS for reward delivery was obtained by analyzing their behavior during the delay period as in our previous study (Paz et al. 2007). Indeed, over time, the animals began licking after the CS, but prior to reward delivery, a phenomenon hereafter termed "anticipatory licking" (see example in Supplemental Fig. 3). Figure 5B plots the rate of anticipatory licking as a function of daily training sessions. The three animals, considered individually or as a group, showed the same trend: an initial period where anticipatory licking rapidly increased followed by a protracted period where anticipatory licking increased more slowly (ANOVA of trials over sessions, $P<0.05$ for all cats). Learning was robust in all three animals. Comparing the difference in licking frequency between the preCS and delay periods between the first and last training sessions with a Mann-Whitney $U$ test yielded significance levels $(P)$ of $0.01,0.02$, and 0.04 for the three animals, respectively.

Overall, these changes in anticipatory licking suggest that memory for the predictive value of the CS is formed gradually over a period of several days. For this reason, the data will be divided in two phases below: an early phase (days 1-3) when the CS is considered to have little, if any predictive value, and a late phase (days 7-9), where the predictive value of the CS is considered to be well learned.

Consistent with this, CS presentation caused a larger increase in theta power at late versus early stages of training (Fig. 5C). This was determined by digitally filtering the LFPs to isolate theta, then rectifying the filtered LFPs with short-windowed (5 msec) RMS, normalizing the data to baseline values, averaging 

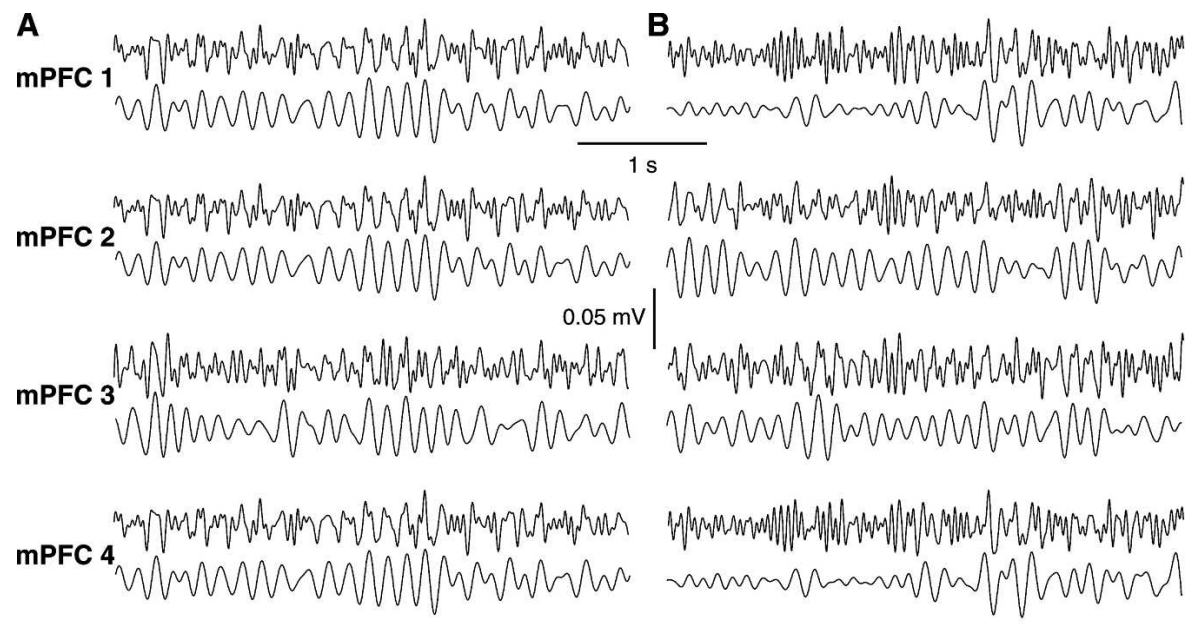

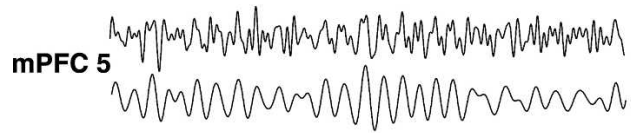
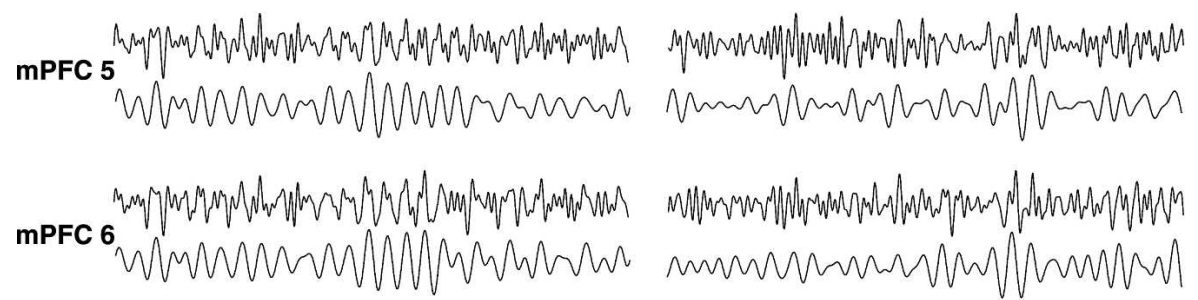

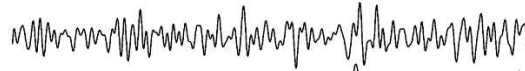

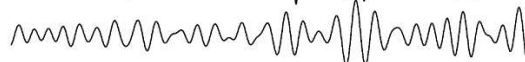
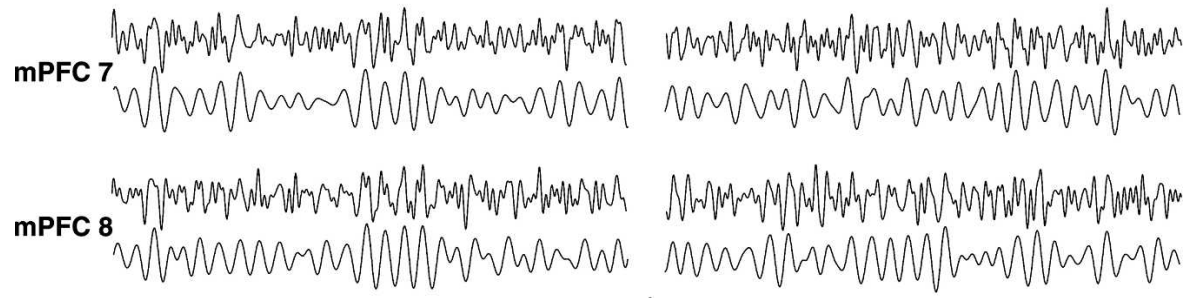

MPFC 1-8
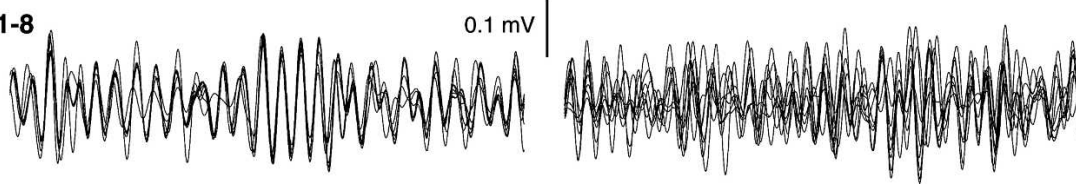

Figure 2. Spontaneous fluctuations in mPFC theta power and synchrony during quiet waking. $(A, B)$ LFPs recorded simultaneously at multiple mPFC sites during two epochs of spontaneous waking. For each recording site (mPFC 1-8), the top and bottom traces, respectively, depict LFPs filtered between 4.5 and $25 \mathrm{~Hz}$ or 4.5 and $10 \mathrm{~Hz}$. An overlay of the eight simultaneously LFPs (digitally filtered between 4.5 and $10 \mathrm{~Hz}$ ) is shown in the bottom of $A$ and $B$. Note how spontaneous theta oscillations could vary from near perfect synchrony to apparent random phase relation independently of fluctuations in amplitude.

separately for early and late stages of learning, and low-pass filtering the result with a cutoff of $6 \mathrm{~Hz}$, resulting in an ongoing measure of theta power. Although the changes in CS-evoked theta power reached significance at early and late stages of training (early, increase of $12.1 \pm 0.9 \%$, paired $t$-test, $P<0.04$; late, increase of $22.9 \pm 1.0 \%$, paired $t$-test, $P<0.001$ ), that seen at the end of the training period were significantly higher ( $t$-test, $P<0.001)$. In contrast, no learning-related change in theta power was seen during the delay ( $t$-test, $P=0.1)$ or reward ( $t$-test, $P=0.5$ ).

It should be mentioned that the learning-related increase in theta power seen during the CS was expressed in the PL and IL regions, considered separately. From early to late stages of learning, the CS-related increase in theta power augmented by $9.1 \pm 1.26 \%$ in IL and by $13.3 \pm 1.89 \%$ in area PL. Both effects remained significant ( $t$-tests, IL, $P<0.01$; PL, $P<0.01$ ), but the difference between the two regions was not ( $t$-tests, $P=0.07$ ).
Because the electrodes were moved $100 \mu \mathrm{m}$ every day during training, it is conceivable that the time-dependent increase in theta power seen in relation to the CS was simply caused by a change in the cortical layers sampled by our electrodes. To control for this possibility, we varied the way the electrodes approached the mPFC such that in two cats (cats 1 and 2) the superficial layers were sampled first and the deep layers second compared with the opposite in the third animal (from deep to superficial). Despite these variations in the sequence of cortical layers recorded during the training paradigm, the same finding was obtained. That is, for all three cats, the CSrelated increase in theta power was significantly higher at late vs. early stages of training ( $t$-tests, $P<0.01$ in the three subjects; for cats $1-3$, increases of $14 \%$ to $27 \%, 10 \%$ to $19 \%$, and $12 \%$ to $25 \%$, respectively). Furthermore, separate ANOVAs on the CS-related increase in theta power are seen early $\left(F_{(2,4887)}=2.13\right)$ or late $\left(F_{(2,4471)}=1.61\right)$ in training confirmed no effect of subjects $(P>0.1$ in both cases).

\section{Learning-related changes in theta synchrony}

Analysis of theta-phase relationships across simultaneously recorded mPFC channels revealed fundamental differences between the theta activity related to the CS at early vs. late stages of learning. To address this question, we relied on the same approach as used above to investigate the impact of theta synchrony on mPFC firing rates (see Fig. 3B). Namely, from each LFP we derived a pure theta-phase signal before and during the CS, averaged this signal across simultaneously recorded mPFC sites, and quantified synchrony by calculating the RMS of the average signal.

As shown in the grand average of Figure 5D, theta oscillations became transiently synchronized following CS onset, particularly at late stages of training. This effect was similarly expressed in the infraand prelimbic regions (from early to late training stages, percent increase of $11.3 \pm 0.98$ and $10.5 \pm 1.25$ for IL and PL regions, respectively). Both effects were significant ( $t$-tests, IL, $P<0.01$; PL, $P<0.01)$, but the difference between the two regions was not ( $t$-test, $P=0.2$ ). However, as shown in the individual examples of Figure 6A-D, there was no constant phase resetting after the CS, only synchronization across channels. Stated otherwise, averaging the data across trials resulted in obliteration or marked reduction of the theta oscillations that were obvious in individual trials. Finally, it should be mentioned that although there was a trend for higher theta synchrony during the delay at late compared with early stages of training, this effect did not reach significance (Mann-Whitney $U$ test, $P=0.07$ ). No learningrelated change in theta synchrony occurred during the reward period. 
Impact of theta synchronization on correlated unit activity in waking and during learning

We next examined how LFP theta synchronization affected the correlation of unit activity at distributed mPFC sites. Because the above LFP analyses revealed learning-related changes in theta power and synchrony in relation to the CS but not the delay or reward, the following section focuses on CS-related activity. To analyze how CS-related theta synchronization affected the correlation of unit activity at distributed $\mathrm{MPFC}$ sites, we computed peri-event histograms (PEHs) of unit firing around theta peaks detected at the same site as the unit activity vs. other mPFC sites at early (days 1-3) and late (days 6-9) stages of learning. The first kind of PEHs, computed with respect to local theta peaks $\left(\mathrm{PEH}_{\mathrm{L}}\right)$ (Fig. 7A1,B1), provides information about the entrainment of firing by local theta activity. The second kind of PEHs, computed with respect to theta peaks detected at $\mathrm{mPFC}$ sites distant from the recorded cell $\left(\mathrm{PEH}_{\mathrm{D}}\right)$ (Fig. 7A2,B2), provides information about the impact of theta synchrony across the mPFC on the entrainment of unit activity. Theta-related rhythmicity in PEHs was quantified by computing RIs as in Figure 4, but on both kinds of PEHs.

As shown in the representative examples of Figure 7, evidence of rhythmic unit firing at the theta frequency was routinely seen in $\mathrm{PEH}_{\mathrm{L}}$ during the CS at early (Fig. 7A1) and late (Fig. 7B1) stages of training. The distributions of RIs derived from the $\mathrm{PEH}_{\mathrm{L}}$ at these two stages of learning were statistically indistinguishable (early, $3.5 \pm 0.58$; late, $3.6 \pm 0.31$ ). In contrast, the
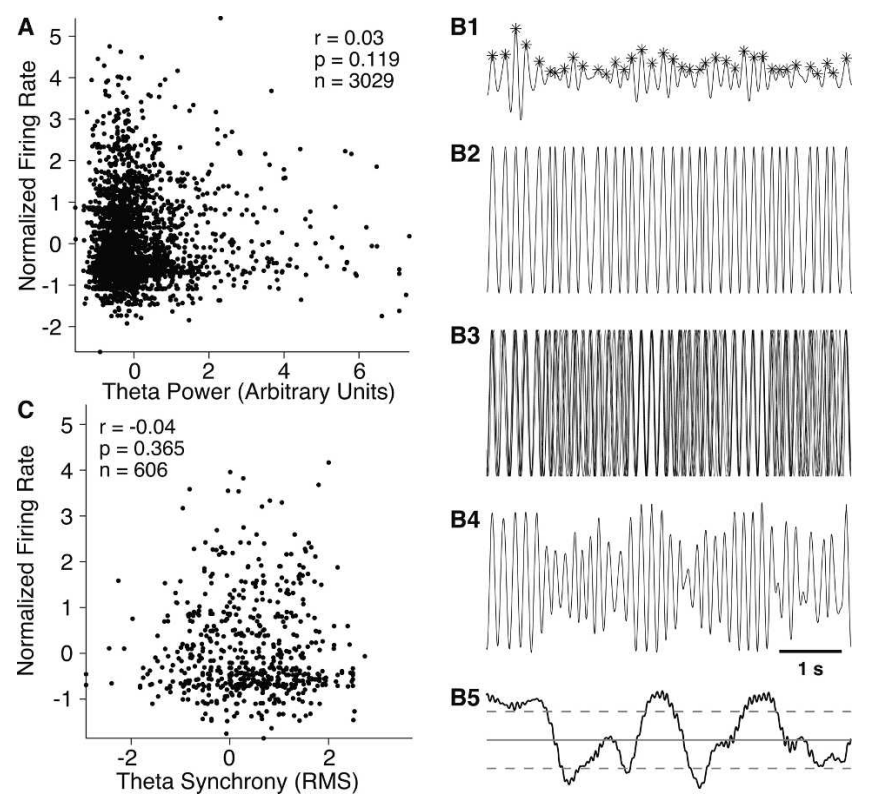

Figure 3. Increased theta power or synchrony is not associated with higher firing rates in mPFC neurons. $(A)$ Firing rates of mPFC neurons ( $Y$-axis, $n=125$, expressed as $Z$-scores) vs. theta power ( $X$-axis) during quiet waking. $(B)$ Method used to quantify theta synchrony at simultaneously recorded $\mathrm{mPFC}$ sites. (B1) In each LFP, theta peaks and troughs were identified. (B2) To extract phase, maxima and minima points were assigned a zero and pi value, the signal was linearly interpolated between them, and then cosined. This created a pure phase signal with no amplitude. (B3) Phase signal for eight simultaneously recorded mPFC sites. The phase information is then averaged across sites (B4) and synchrony quantified by computing the root mean square (RMS) of the averaged signal (B5). Dashed lines show 2.5\% confidence intervals obtained by computing mean and SD over a $1-\mathrm{h}$ recording period. (C) mPFC firing rates $(Y$-axis) vs. theta synchrony ( $X$-axis) during quiet waking. The values of theta synchrony are expressed as Z-scores in order to allow plotting of all the cells together in one figure.

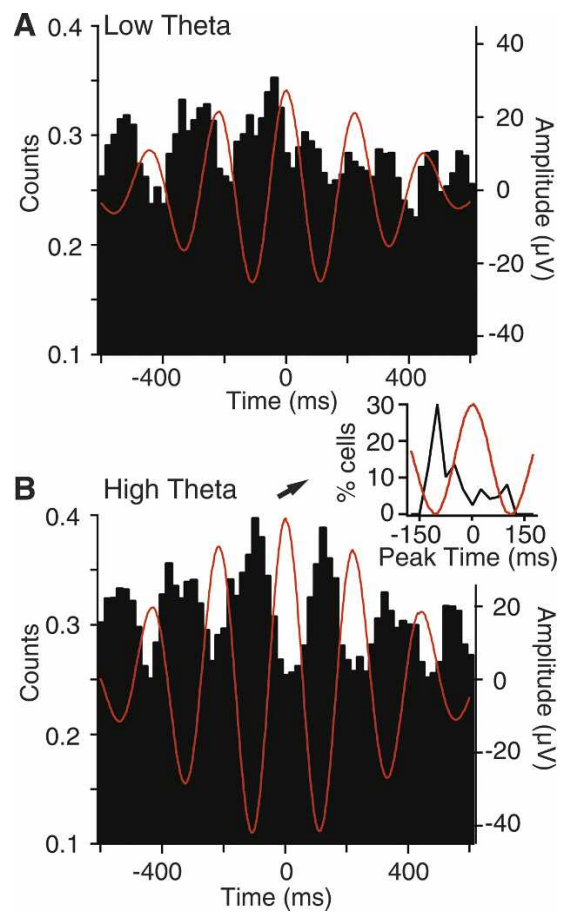

Figure 4. Increased theta power is associated with enhanced firing rhythmicity. Peri-event histograms of unit discharges ( $Y$-axis) around theta peaks (zero on $X$-axis) in periods of low $(A)$ or high $(B)$ theta power. These population histograms were computed from a subset of $66 \mathrm{mPFC}$ cells that had $\mathrm{PEH}$ peak times in the -75 to $-125 \mathrm{msec}$ range during periods of high theta power. Inset in $B$ shows frequency distribution of peak times (black curve) in our sample of mPFC cells. In a majority of cells, the $\mathrm{PEH}$ peak coincided with the negative phase of the local theta.

$\mathrm{PEH}_{\mathrm{D}}$, computed for the same cells, but around theta peaks detected at other mPFC sites, displayed stronger evidence of thetarelated activity at late (Fig. 7B2) than early (Fig. 7A2) stages of learning. Repeating this analysis for all available mPFC cells confirmed that entrainment by distant theta was significantly higher at late vs. early stages of training (RIs of $3.3 \pm 0.15$ and $4.1 \pm 0.14$ for early vs. late stages of training; $t$-test, $P<0.002$ ). Separately computing RIs for IL vs. PL cells using theta peaks detected in PL and IL, respectively, yielded similarly high RIs at late stages of training (IL, $4.4 \pm 0.2$; PL, $3.9 \pm 0.3$ ) with no significant difference between the two sets of neurons ( $t$-test, $P>0.1)$. This is an important observation because it implies that the activity of these two prefrontal regions is synchronized during learning.

Finally, it should be noted that the difference between the entrainment of unit activity by distant theta at early vs. late phases of training persisted after we normalized the distant RIs by the local RIs. As shown in the frequency distribution of normalized distant RIs (Fig. 7C), this analysis confirmed that the entrainment of mPFC unit firing by distant theta was significantly higher at late (Fig. 7C, red) compared with early (Fig. 7C, black) stages of training.

\section{Discussion}

The present study was undertaken to examine the impact of theta oscillations on the synchronization of neuronal activity at distributed mPFC sites. The interest of this issue derives from earlier data suggesting that, in a trace-conditioning task, synchronized mPFC activity can facilitate hippocampo-neocortical communication by enhancing entorhinal to perirhinal transfer (Paz et al. 2007). However, the origin of this enhanced CS-related 
A
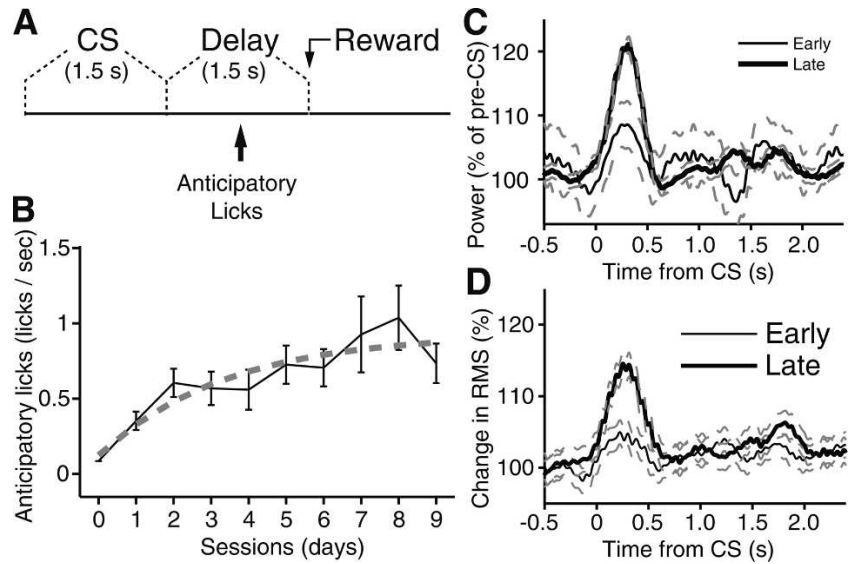

Figure 5. Learning-related increase in theta synchrony during the CS. Learning progression in an appetitive trace-conditioning paradigm and related changes in $\mathrm{mPFC}$ theta power. $(A)$ Scheme showing the relative timing of the visual CS, delay period, and reward delivery in the traceconditioning paradigm used here. To assess learning, we measured the rate of anticipatory licking during the delay period. (B) Graph plotting the rate of anticipatory licking ( $Y$-axis) as a function of training sessions ( $X$ axis). (C) Graph plotting theta power ( $Y$-axis) as a function of time $(X$ axis) around CS onset (time zero) at early (thin line) and late (thick lines) stages of learning. The data was normalized to pre-CS values. Dashed lines indicate standard error. To generate this figure, each channel was digitally filtered to isolate theta. Then, the signals were rectified with short-windowed (5 msec) RMS, normalized to baseline values, averaged for early/late, and low-pass filtered with a cutoff of $6 \mathrm{~Hz}$, resulting in an ongoing measure of theta power. $(D)$ Graph plotting theta synchrony (RMS, $Y$-axis) as a function of time ( $X$-axis) around CS onset (time zero) at early (thin line) and late (thick lines) stages of learning. The data was normalized to pre-CS values. Dashed lines indicate standard error.

mPFC synchrony was unknown. Here, we show that theta oscillations play a key role in synchronizing the activity of mPFC cells. Indeed, mPFC theta power increased from early to late stages of learning during the CS. Moreover, at late stages of training, CS onset caused a rapid phase locking of theta activity at distributed $\mathrm{mPFC}$ sites, entraining $\mathrm{mPFC}$ neurons to fire in a rhythmic and synchronized manner. In the following, we consider the significance of these findings for the role of the mPFC in memory consolidation.

\section{Intricate connectivity between the mPFC, hippocampus, neocortex, and rhinal cortices}

As reviewed above, multiple lines of evidence suggest that the mPFC and hippocampus have an opposite temporal profile of involvement in memory consolidation. Indeed, lesion (ZolaMorgan and Squire 1986; Kim and Fanselow 1992; Takehara et al. 2003; Maviel et al. 2004), molecular (Frankland et al. 2004), imaging (Takashima et al. 2006), and pharmaco-behavioral (Takehara-Nishiuchi et al. 2006) studies all converge to suggest that shortly after training, memories are heavily dependent on the hippocampus, but not the mPFC. Later on, however, they gradually become independent of the hippocampus and, in parallel, dependent on the mPFC. During this transition, it is thought that transient hippocampal traces are gradually transferred to the neocortex for long-term storage (Squire and Alvarez 1995; Buzsaki 1996; Sutherland and McNaughton 2000) and that the mPFC might facilitate this process (Frankland and Bontempi 2005; Takashima et al. 2006).

What is the anatomical substrate of these interactions? Tract-tracing studies have revealed that the mPFC has closer ties with the hippocampus than most neocortical areas. Indeed, whereas the mPFC receives direct inputs from the temporal hippocampus (Swanson 1981; Ferino et al. 1987; Jay and Witter
1991; Thierry et al. 2000), the connections between the hippocampus and most of the neocortex are indirect, involving two or more synaptic relays through the rhinal cortices (Burwell and Witter 2002). Yet, the mPFC is in a position to influence interactions between the hippocampus and neocortex because it projects to the rhinal cortices (Room et al. 1985; Sesack et al. 1989; Hurley et al. 1991).

Clues as to the significance of this projection come from a consideration of the ability of the rhinal cortices to shuttle information between the neocortex and hippocampus (for review, see de Curtis and Pare 2004). In baseline conditions in vivo and in vitro, rhinal transfer of hippocampal and neocortical activity occurs with a low probability (Biella et al. 2002; Pelletier et al. 2004; Paz et al. 2006), because there are strong inhibitory pressures in this pathway (Biella et al. 2001; Martina et al. 2001) including local and long-range feedforward inhibition (Pinto et al. 2006; Apergis-Schoute et al. 2007). However, as discussed below, we have recently shown that in some circumstances these inhibitory pressures can be overcome by extrinsic glutamatergic afferents from the mPFC.

\section{Impact of synchronized mPFC activity on hippocampal-neocortical communication via the rhinal cortices}

It is well known that during the acquisition of trace-conditioning tasks, the activity profile of hippocampal and mPFC neurons changes (Berger et al. 1983; Solomon et al. 1986; Weiss et al. 1996; Baeg et al. 2001; Munera et al. 2001; McEchron et al. 2003; Gilmartin and McEchron 2005). In a previous study relying on the same trace-conditioning task (Paz et al. 2007), we found that as the CS gained predictive value, the proportion of CSresponsive mPFC neurons significantly increased, but that the majority of mPFC cells remained CS unresponsive. Nevertheless, when we examined the impact of mPFC spikes on correlated firing among rhinal neurons, mPFC activity was found to facilitate entorhinal to perirhinal communication. Remarkably, this effect was not linked to an increase in the firing rate of mPFC cells during the CS, but mostly to an increase in firing synchrony that was observed even among mPFC cells that were not CSresponsive (Paz et al. 2007).

The significance of these findings stems from the fact that the entorhinal-to-perirhinal pathway is a necessary link between the hippocampus and neocortex. As a result, the contribution of the mPFC to memory consolidation might, in part at least, reside in its ability to facilitate the transfer of hippocampal activity to the neocortex by enhancing entorhinal to perirhinal communication. Thus, shedding light on the mechanisms underlying this increased $\mathrm{mPFC}$ synchrony is an issue of critical importance. Because the temporal hippocampus sends a strong projection to the mPFC, theta oscillations emerged as an obvious candidate mechanism for synchronizing mPFC activity. Indeed, earlier studies had revealed that hippocampal theta could entrain the firing of mPFC cells (Jones and Wilson 2005a,b; Siapas et al. 2005). In support of the idea that theta oscillations play a critical role in synchronizing mPFC activity during learning, we observed that mPFC theta power increased during the CS and that this effect became stronger late in training, as the CS gained predictive value for reward delivery. Moreover, at late, but not early stages of learning, CS onset caused a transient phase locking of theta oscillations at distributed mPFC sites, entraining mPFC cells to fire rhythmically and synchronously with the theta oscillations. Thus, these results raise the intriguing possibility that the hippocampus, by generating a stronger theta source during learning, can help to synchronize mPFC activity, in turn facilitating rhinal transfer of its activity to the neocortex. 


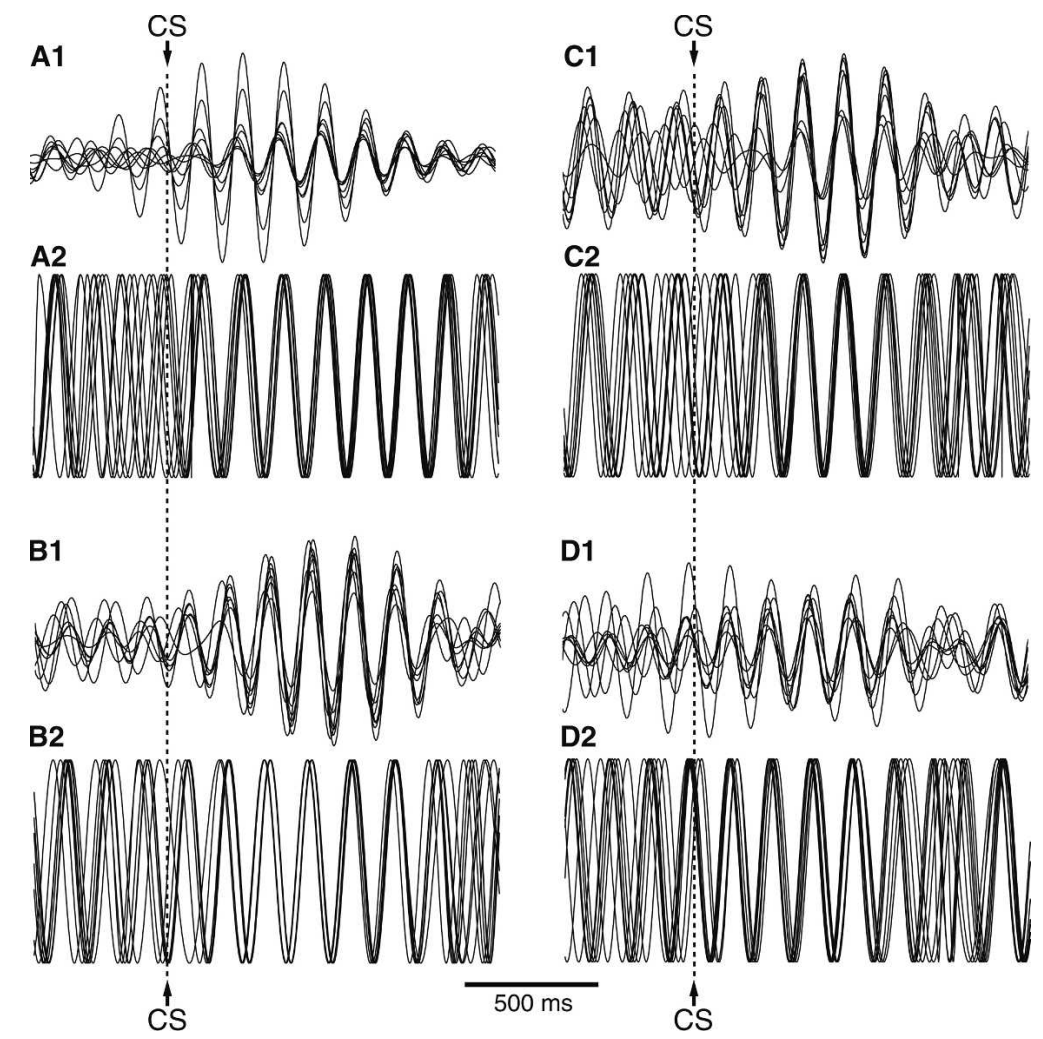

Figure 6. At late stages of learning, the CS causes a transient phase locking of theta activity in the mPFC. (A-D) Fluctuations in theta activity (1) and synchrony (2) during CS presentation at late stages of learning. In each case, the top traces (1) show an overlay of eight LFPs digitally filtered at the theta frequency, whereas the bottom traces (2) show phase information extracted as in Figure 3B. The data was obtained on the training session number 8 (next to last training session) in two different animals (left and right columns, respectively).

However, lesion studies suggest that the time window during which both the hippocampus and $\mathrm{mPFC}$ are required for memory consolidation is short lived. At long post-training delays, the hippocampus is no longer involved. Thus, the theta- related synchronization of mPFC activity and the consequent facilitation of rhinal interactions appear to constitute a transitory phase. It is possible that during this period the rhythmic thetarelated firing of mPFC cells not only facilitates rhinal transfer of hippocampal traces to the neocortex but helps strengthen synaptic links between spatially distributed cortical neurons representing different components of the experience to be remembered. Such an action of mPFC neurons could be mediated by their widespread cortical projections (Room et al. 1985; Sesack et al. 1989; Hurley et al. 1991). Later on, when hippocampal activity is no longer required because the links between relevant cortical cell assemblies are stronger, the mPFC might contribute to maintain and stabilize these connections. Another possibility, not exclusive of the previous scenario, is that the MPFC then becomes critical to the reactivation of the distributed neocortical storage sites when memories are recalled (see Frankland and Bontempi 2005).

\section{Conclusions}

Our results suggest that theta oscillations contribute to synchronize the activity of distributed mPFC sites entraining $\mathrm{mPFC}$ neurons to fire rhythmically and synchronously. In a learning context, this effect promotes rhinal transfer of hippocampal traces to the neocortex. We speculate that the theta-related rhythmic firing of mPFC cells might also assist in strengthening the links between spatially distributed cortical neurons representing different components of the experience to be remembered.
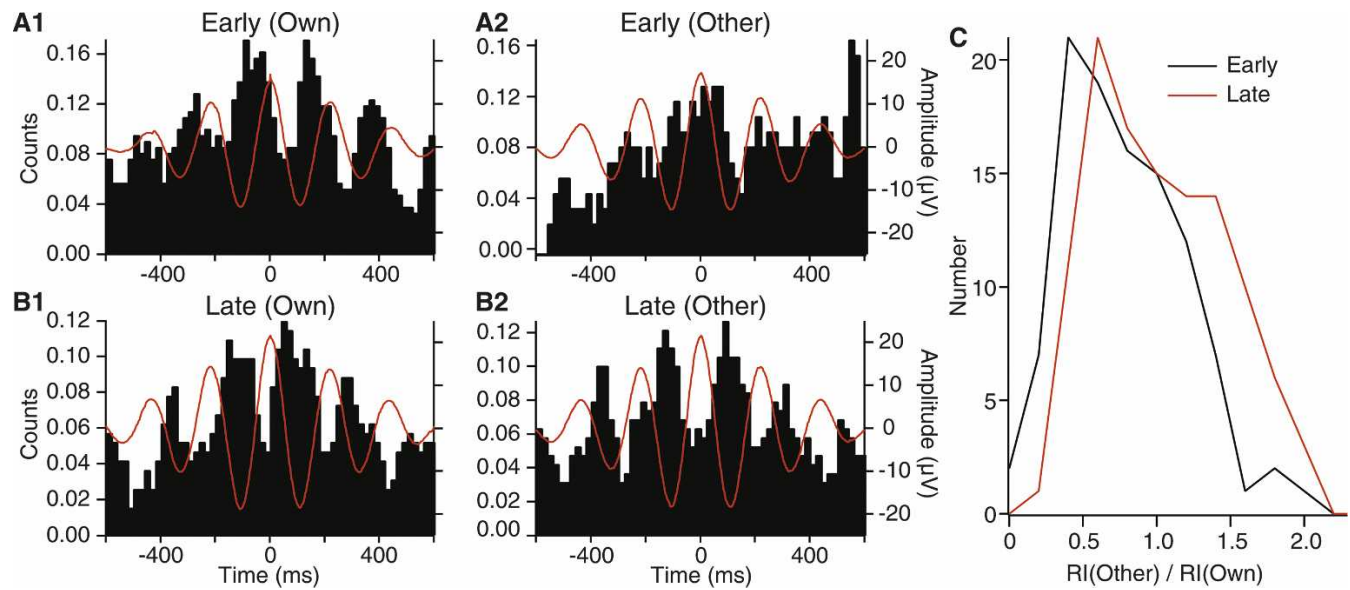

Figure 7. Learning-dependent theta synchronization of unit activity at multiple mPFC sites during the CS. ( $A, B$ ) Peri-event histograms (PEHs) of unit discharges ( $Y$-axis) around theta peaks during the $C S$ at early $(A)$ or late $(B)$ stages of training for a representative cell. Theta peaks were detected using the theta activity recorded from the same (1, Own) or different (2, Other) electrodes than those used to record unit activity. (C) Frequency distribution of rhythmicity indices (RIs) during the CS at early (black line) vs. late stages (red line) of training. The Rls were obtained from PEHs computed around theta peaks detected on channels other than the one used to record the unit. The data was normalized to the RI observed when the PEHs were computed using theta peaks detected on the same electrode as that used to record the unit activity. The frequency distribution show the normalized RIs of 15 (early) and 16 (late) MPFC cells around the theta peaks detected in the LFPs recorded at seven other mPFC sites than where each cell was recorded. 


\section{Materials and Methods}

\section{Surgery}

Three adult cats were implanted with an array of high-impedance microelectrodes aimed at the mPFC (Fig. 1A). These are the same cats and recordings as used in our previous study (Paz et al. 2007); however, the present report focuses on the theta modulation of mPFC activity, an issue that was not explored in our earlier study. The experiments were performed in cats because the comparatively large size of the cat brain facilitates the accurate placement of many microelectrodes in multiple brain regions.

All procedures were approved by the Institutional Animal Care and Use Committee of Rutgers University, in compliance with the Guide for the Care and Use of Laboratory Animals (Department of Health and Human Services). Cats were preanesthetized with ketamine and xylazine (15 and $2 \mathrm{mg} / \mathrm{kg}$, i.m.) and artificially ventilated with a mixture of ambient air, oxygen, and isoflurane. Atropine $(0.05 \mathrm{mg} / \mathrm{kg}$, i.m.) was administered. The end-tidal $\mathrm{CO}_{2}$ concentration was maintained at $3.7 \% \pm 0.2 \%$ and the body temperature at $37-38^{\circ} \mathrm{C}$ with a heating pad. Bupivacaine (s.c.) was administered in the region to be incised $15 \mathrm{~min}$ prior to the first incision. In sterile conditions, an incision was performed on the midline of the scalp and the skull muscles were retracted. Then, a screw was inserted in the skull overlying the cerebellum and acted as the reference. In addition, four screws were cemented to the skull to later fix the cat's head without pain or pressure. Finally, after trepanation and opening of the dura mater, an array of nine high-impedance tungsten microelectrodes (10-12 M $\Omega$; Frederic Haer Co.) was stereotaxically lowered with an oblique, lateromedial approach until the electrodes reached the mPFC. Three of the electrodes were aimed to the infralimbic region and the others to the prelimbic region. Here, it should be mentioned that that the connectivity of the pre- and infralimbic areas is similar in rats and cats (for example, see Krettek and Price 1977; McDonald 1998).

At the end of the surgery, the animals were administered penicillin (20,000 UI/kg, i.m.) and an analgesic (Ketophen, $2 \mathrm{mg} /$ $\mathrm{kg}$, s.c. daily for $3 \mathrm{~d}$ ). Recording sessions began $8 \mathrm{~d}$ after the surgery.

\section{Behavior}

The learning task was an appetitive trace-conditioning paradigm, where a visual conditioned stimulus (CS; $1.5 \mathrm{sec}$ ) was followed by a 1.5-sec delay period, after which a liquid reward was administered. Trials occurred at random intervals of 30-90 sec. The animals were subjected to around 50 trace-conditioning trials (median 55.5) per daily session for nine consecutive days. The liquid reward $(2 \mathrm{~mL} /$ trial) was a preferred food (Gerber's pureed baby food "sweet potatoes and turkey"). The animals were only fed during recording sessions, and their weight was monitored daily to maintain body weight within $10 \%$ of its initial value.

Behavior was monitored by means of a switch detecting when the tongue of the animals contacted the receptacle where the food reward was administered. The visual CS was a global change in the illumination (from black to white) of a 12 " LCD screen placed one foot in from of the animals. Detection of the visual CS did not necessitate that the animals maintain a fixed gaze at the center of the LCD screen because it encompassed most of their visual field. Because the animals were hungry, they were highly aroused and remained awake at all times (as assessed by EEG recordings) with their eyes opened.

\section{Histology}

At the end of the experiments, the animals were given an overdose of sodium pentobarbital (50 mg/kg, i.v.) and perfused-fixed. The recording sites were marked with electrolytic lesions $(0.5$ $\mathrm{mA}, 5 \mathrm{sec}$; Fig. 1B, arrows). The brains were later sectioned on a vibrating microtome (at $100 \mu \mathrm{m}$ ) and stained with cresyl violet to verify the position of recording electrodes. Microelectrode tracks were reconstructed by combining micrometer readings with the histology.

\section{Recording and analysis methods}

Our previous experience with the present recording method has taught us that while unit recordings are stable within a recording session (when the animals are head restrained), we generally lose the cells in between sessions, when the animals are free to move. To circumvent this problem, the electrodes were moved down $100 \mu \mathrm{m}$ each day. To ensure mechanical stability, we waited 30 min after moving the electrodes before beginning the recordings. The signals picked up by the electrodes were observed on an oscilloscope, digitized, and stored on a hard disk. Spike sorting was performed off-line using custom-made software that features a clustering algorithm based on principal component analysis and K-means. Examples of clustering are provided in Supplemental Figure 1.

\section{Computation of rhythmicity indices}

We computed peri-event histograms (PEHs) of mPFC unit discharges using the positive peaks of theta LFP oscillations as temporal references (see Supplemental Fig. 2). To this end, field potentials were digitally filtered to isolate theta oscillations (FIR digital filter of order 251; matlab fir 1 and filtfilt functions for zero phase distortion; $4.5-10 \mathrm{~Hz}$ ). Theta cycles with positive peak $\geq 2$ $\mathrm{SD}$ of the overall filtered signal were identified (Supplemental Fig. 2A). Then, PEHs of unit firing were computed around the positive peaks of the theta oscillations (Supplemental Fig. 2C). Depending on the analysis, the field and unit recordings were obtained from the same or different electrodes. A rhythmicity index (RI) was computed to quantify the strength of the modulation of unit activity by theta oscillations. To compute RIs, we measured the maximal peak to trough difference found within half a cycle of the three central peaks (six measures total), averaged these values, and divided the result by the mean of the entire histogram to normalize for firing rate (Supplemental Fig. 2C). To determine whether unit activity was significantly modulated by theta oscillations, we constructed 100 PEHs of unit discharges using the same spike train, but with respect to random times. The original RI was then compared with the distribution of random RIs. The actual PEH was considered significant if its RI was higher than $95 \%$ of the RIs based on randomly generated PEHs.

\section{Quantification of theta synchrony}

To assess theta synchrony across multiple simultaneously recorded mPFC sites, the following approach was used: after digital filtering as above, theta peaks and troughs were identified in each LFP by taking the derivative of the filtered signal and identifying change in sign points. These maxima and minima points were assigned zero and pi values. Then, the signal was linearly interpolated between them and cosined. This created a pure phase signal with standardized amplitude varying between -1 and 1 . The phase signal derived from each simultaneously recorded LFP was then averaged across channels. Because these are pure phase signals, moment-to-moment variations in amplitude seen as a result averaging reflect changes in theta synchrony across the simultaneously recorded mPFC sites. For instance, in conditions of low synchrony, the different channels cancel each other and averaging results in a low-amplitude signal. In contrast, averaging channels with synchronized theta results in a high-amplitude signal. Synchrony was quantified by computing the RMS of the average signal (by filtering the squared signal with a Kaiser window of $150 \mathrm{msec}$ and extracting its root). All values are reported as mean \pm SEM. The validity of this phase analysis was verified by direct extraction of phase using FFTs. Both approaches yielded qualitatively identical results.

\section{Acknowledgments}

This material is based upon work supported by NIMH grant R01MH-073610 and NRSA grant 5F32MH076640. 


\section{References}

Apergis-Schoute, J., Pinto, A., and Pare, D. 2006. Ultrastructural organization of medial prefrontal inputs to the rhinal cortices. Eur. J. Neurosci. 24: 135-144.

Apergis-Schoute, J., Pinto, A., and Pare, D. 2007. Muscarinic control of long-range GABAergic inhibition within the rhinal cortices. $J$. Neurosci. 27: 4061-4071.

Baeg, E.H., Kim, Y.B., Jang, J., Kim, H.T., Mook-Jung, I., and Jung, M.W. 2001. Fast spiking and regular spiking neural correlates of fear conditioning in the medial prefrontal cortex of the rat. Cereb. Cortex 11: $441-451$.

Barbas, H. and Blatt, G.J. 1995. Topographically specific hippocampal projections target functionally distinct prefrontal areas in the rhesus monkey. Hippocampus 5: 511-533.

Berger, T.W., Rinaldi, P.C., Weisz, D.J., and Thompson, R.F. 1983. Single-unit analysis of different hippocampal cell types during classical conditioning of rabbit nictitating membrane response. $J$. Neurophysiol. 50: 1197-1219.

Biella, G., Uva, L., and de Curtis, M. 2001. Network activity evoked by neocortical stimulation in area 36 of the guinea pig perirhinal cortex. J. Neurophysiol. 86: 164-172.

Biella, G., Uva, L., and de Curtis, M. 2002. Propagation of neuronal activity along the neocortical-perirhinal-entorhinal pathway in the guinea pig. J. Neurosci. 22: 9972-9979.

Bontempi, B., Laurent-Demir, C., Destrade, C., and Jaffard, R. 1999. Time-dependent reorganization of brain circuitry underlying long-term memory storage. Nature 400: 671-675.

Burwell, R.D. and Witter, M.P. 2002. Basic anatomy of the parahippocampal region in monkeys and rats. In The parahippocampal region (eds. M.P. Witter and F. Wouterlood), pp. 53-59. Oxford University Press, New York.

Buzsaki, G. 1996. The hippocampo-neocortical dialogue. Cereb. Cortex 6: 81-92.

de Curtis, M. and Pare, D. 2004. The rhinal cortices: A wall of inhibition between the neocortex and the hippocampus. Prog. Neurobiol. 74: $101-110$

Ferino, F., Thierry, A.M., and Glowinski, J. 1987. Anatomical and electrophysiological evidence for a direct projection from Ammon's horn to the medial prefrontal cortex in the rat. Exp. Brain Res. 65: $421-426$.

Frankland, P.W. and Bontempi, B. 2005. The organization of recent and remote memories. Nat. Rev. Neurosci. 6: 119-130.

Frankland, P.W., Bontempi, B., Talton, L.E., Kaczmarek, L., and Silva, A.J. 2004. The involvement of the anterior cingulate cortex in remote contextual fear memory. Science 304: 881-883.

Gilmartin, M.R. and McEchron, M.D. 2005. Single neurons in the medial prefrontal cortex of the rat exhibit tonic and phasic coding during trace fear conditioning. Behav. Neurosci. 119: 1496-1510.

Hirose, S., Ino, T., Takada, M., Kimura, J., Akiguchi, I., and Mizuno, N. 1992. Topographic projections from the subiculum to the limbic regions of the medial frontal cortex in the cat. Neurosci. Lett. 139: 61-64.

Hurley, K.M., Herbert, H., Moga, M.M., and Saper, C.B. 1991. Efferent projections of the infralimbic cortex of the rat. J. Comp. Neurol. 308: 249-276.

Irle, E. and Markowitsch, H.J. 1982. Widespread cortical projections of the hippocampal formation in the cat. Neuroscience 7: 2637-2647.

Jay, T.M. and Witter, M.P. 1991. Distribution of hippocampal CA1 and subicular efferents in the prefrontal cortex of the rat studied by means of anterograde transport of Phaseolus vulgaris-leucoagglutinin. J. Comp. Neurol. 313: 574-586.

Jones, M.W. and Wilson, M.A. 2005a. Theta rhythms coordinate hippocampal-prefrontal interactions in a spatial memory task. PLoS Biol. 3: e402. doi: 10.1371/journal.pbio.0030402.

Jones, M.W. and Wilson, M.A. 2005b. Phase precession of medial prefrontal cortical activity relative to the hippocampal theta rhythm. Hippocampus 15: 867-873.

Kim, J.J. and Fanselow, M.S. 1992. Modality-specific retrograde amnesia of fear. Science 256: 675-677.

Krettek, J.E. and Price, J.L. 1977. Projections from the amygdaloid complex to the cerebral cortex and thalamus in the rat and cat. $J$. Comp. Neurol. 172: 687-722.

Martina, M., Royer, S., and Pare, D. 2001. Propagation of neocortical inputs in the perirhinal cortex. J. Neurosci. 21: 2878-2888.
Maviel, T., Durkin, T.P., Menzaghi, F., and Bontempi, B. 2004. Sites of neocortical reorganization critical for remote spatial memory. Science 305: $96-99$.

McDonald, A.J. 1998. Cortical pathways to the mammalian amygdala. Prog. Neurobiol. 55: 257-332.

McEchron, M.D., Tseng, W., and Disterhoft, J.F. 2003. Single neurons in CA1 hippocampus encode trace interval duration during trace heart rate (fear) conditioning in rabbit. J. Neurosci. 23: 1535-1547.

Munera, A., Gruart, A., Munoz, M.D., Fernandez-Mas, R., and Delgado-Garcia, J.M. 2001. Hippocampal pyramidal cell activity encodes conditioned stimulus predictive value during classical conditioning in alert cats. J. Neurophysiol. 86: 2571-2582.

Paz, R., Pelletier, J.G., Bauer, E.P., and Pare, D. 2006. Emotional enhancement of memory via amygdala-driven facilitation of rhinal interactions. Nat. Neurosci. 9: 1321-1329.

Paz, R., Bauer, E.P., and Pare, D. 2007. Learning-related facilitation of rhinal interactions by medial prefrontal inputs. J. Neurosci. 27: 6542-6551.

Pelletier, J.G., Apergis, J., and Pare, D. 2004. Low-probability transmission of neocortical and entorhinal impulses through the perirhinal cortex. J. Neurophysiol. 91: 2079-2089.

Pinto, A., Fuentes, C., and Pare, D. 2006. Feedforward inhibition regulates perirhinal transmission of neocortical inputs to the entorhinal cortex: Ultrastructural study in guinea pigs. J. Comp. Neurol. 495: 722-734.

Room, P., Russchen, F.T., Groenewegen, H.J., and Lohman, A.H. 1985. Efferent connections of the prelimbic (area 32) and the infralimbic (area 25) cortices: An anterograde tracing study in the cat. J. Comp. Neurol. 242: 40-55.

Sesack, S.R., Deutch, A.Y., Roth, R.H., and Bunney, B.S. 1989. Topographical organization of the efferent projections of the medial prefrontal cortex in the rat: An anterograde tract-tracing study with Phaseolus vulgaris leucoagglutinin. J. Comp. Neurol. 290: 213-242.

Siapas, A.G., Lubenov, E.V., and Wilson, M.A. 2005. Prefrontal phase locking to hippocampal theta oscillations. Neuron 46: 141-151.

Solomon, P.R., Vander Schaaf, E.R., Thompson, R.F., and Weisz, D.J. 1986. Hippocampus and trace conditioning of the rabbit's classically conditioned nictitating membrane response. Behav. Neurosci. 100: 729-744.

Squire, L.R. and Alvarez, P. 1995. Retrograde amnesia and memory consolidation: A neurobiological perspective. Curr. Opin. Neurobiol. 5: 169-177.

Sutherland, G.R. and McNaughton, B. 2000. Memory trace reactivation in hippocampal and neocortical neuronal ensembles. Curr. Opin. Neurobiol. 10: 180-186.

Swanson, L.W. 1981. A direct projection from Ammon's horn to prefrontal cortex in the rat. Brain Res. 217: 150-154.

Takashima, A., Petersson, K.M., Rutters, F., Tendolkar, I., Jensen, O., Zwarts, M.J., McNaughton, B.L., and Fernandez, G. 2006. Declarative memory consolidation in humans: A prospective functional magnetic resonance imaging study. Proc. Natl. Acad. Sci. 103: 756-761.

Takehara, K., Kawahara, S., and Kirino, Y. 2003. Time-dependent reorganization of the brain components underlying memory retention in trace eyeblink conditioning. J. Neurosci. 23: 9897-9905.

Takehara-Nishiuchi, K., Nakao, K., Kawahara, S., Matsuki, N., and Kirino, Y. 2006. Systems consolidation requires postlearning activation of NMDA receptors in the medial prefrontal cortex in trace eyeblink conditioning. J. Neurosci. 26: 5049-5058.

Thierry, A.M., Gioanni, Y., Degenetais, E., and Glowinski, J. 2000. Hippocampo-prefrontal cortex pathway: Anatomical and electrophysiological characteristics. Hippocampus 10: 411-419.

Weiss, C., Kronforst-Collins, M.A., and Disterhoft, J.F. 1996. Activity of hippocampal pyramidal neurons during trace eyeblink conditioning. Hippocampus 6: 192-209.

Wiltgen, B.J., Brown, R.A., Talton, L.E., and Silva, A.J. 2004. New circuits for old memories: The role of the neocortex in consolidation. Neuron 44: $101-108$.

Zola-Morgan, S. and Squire, L.R. 1986. Memory impairment in monkeys following lesions limited to the hippocampus. Behav. Neurosci. 100: $155-160$.

Received January 25, 2008; accepted in revised form April 24, 2008. 


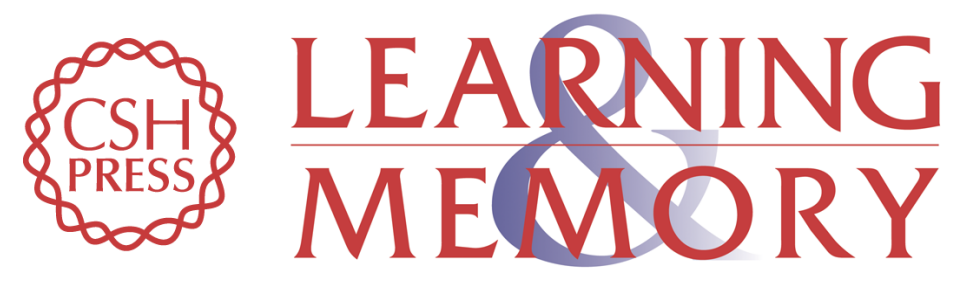

\section{Theta synchronizes the activity of medial prefrontal neurons during learning}

Rony Paz, Elizabeth P. Bauer and Denis Paré

Learn. Mem. 2008, 15:

Access the most recent version at doi:10.1101//m.932408

References This article cites 45 articles, 11 of which can be accessed free at:

http://learnmem.cshlp.org/content/15/7/524.full.html\#ref-list-1

License

Email Alerting Receive free email alerts when new articles cite this article - sign up in the box at the Service top right corner of the article or click here. 